\title{
Percepção da população sobre o papel do Farmacêutico no contexto da pandemia do
} novo coronavírus

\author{
Population perception of the role of the Pharmacist in the context of the new coronavirus pandemic
}

Percepción de la población sobre el papel del Farmacéutico en el contexto de la nueva pandemia de coronavirus

\section{Resumo}

O objetivo deste artigo é avaliar a percepção da população sobre o papel do farmacêutico no contexto da pandemia do novo coronavírus. Trata-se de um estudo descritivo exploratório de abordagem quantitativa realizado, via plataforma Google Formulários. Foram incluídas pessoas de ambos os sexos, maiores de 18 anos, residentes no Brasil. O link do questionário foi enviado pelo WhatsApp, Instagram, Facebook e e-mail. Dos 1.748 questionários respondidos, 64,5\% eram de mulheres, a média de idade dos participantes foi de $25,3 \pm 7,9$ anos e a maioria se declarou parda $(56,8 \%)$. Do total de participantes, $96,5 \%$ consideraram o farmacêutico profissional da saúde, no entanto 53,4\% não foram atendidos por ele durante a pandemia e $68,6 \%$ não buscaram orientação deste. Constatou-se que a população deste estudo reconheceu o farmacêutico como profissional de saúde na linha de frente contra o coronavírus, porém não sabia identificar seu papel diante da pandemia.

Palavras-chave: Coronavírus; Pandemia; Farmacêutico; Assistência farmacêutica.

\begin{abstract}
Absctract
The aim of this article is to assess the population's perception of the role of the pharmacist in the context of the new coronavirus pandemic. This is a descriptive exploratory study with a quantitative approach carried out, via the Google Forms platform. People of both sexes, over 18 years of age, residing in Brazil were included. The questionnaire link was sent by Whatsapp, Instagram, Facebook and email. Of the 1,748 questionnaires answered were from women (64.5\%), the average age of the participants was $25.3 \pm 7.9$ years and the majority was brown (56.8\%). Almost all 96.5\% considered the pharmacist to be a health professional, however $53.4 \%$ were not attended by him during the pandemic and $68.6 \%$ did not seek guidance from him. It was found that the population of this study recognized the pharmacist as a health professional on the front line against the coronavirus, but did not know how to identify his role in the face of the pandemic.
\end{abstract}

Keywords: Coronavirus; Pandemics; Pharmaceutic; Pharmaceutical services.

\section{Resumen}

El propósito de este artículo es evaluar la percepción que tiene la población sobre el papel del farmacéutico en el contexto de la nueva pandemia de coronavirus. Se trata de un estudio exploratorio descriptivo con enfoque cuantitativo realizado, a través de la plataforma Google Forms. Se incluyeron personas de ambos sexos, mayores de 18 años, residentes en Brasil. El enlace del cuestionario fue enviado por Whatsapp, Instagram, Facebook y correo electrónico. De los 1.748 cuestionarios respondidos fueron de mujeres $(64,5 \%)$, la edad media de los participantes fue de 25,3 $\pm 7,9$ años y la mayoría era morena $(56,8 \%)$. Casi todo el $96,5 \%$ consideró al farmacéutico como un profesional de la salud, sin embargo el 53,4\% no fue atendido por él durante la pandemia y el 68,6\% no buscó su orientación. Se constató que la población de este estudio reconoció al farmacéutico como un profesional de la salud en primera línea contra el coronavirus, pero no supo identificar su papel ante la pandemia.

Palabras-clave: Coronavirus; Pandemia; Farmacéutico; Cuidado farmacéutico. 


\section{Introdução}

O SARS-CoV-2 é um RNA vírus que causa infecções no trato respiratório, e dentre os principais sinais e sintomas da COVID-19 destacam-se: febre, tosse seca, dispneia e diarreia, nos casos mais brandos da doença. A transmissão ocorre por meio de gotículas produzidas quando um indivíduo infectado tosse ou respira (Ashour e colaboradores, 2020). Já nos casos graves, a doença manifesta-se por pneumonia, acidose metabólica, choque séptico e sangramento. Há também os fatores que predispõem a doença e sua forma mais grave, como o contato com indivíduos que foram contaminados, viagem para áreas afetadas 14 dias antes do início dos sintomas, idosos (+65 anos), e comorbidades como a hipertensão (Dzingirai e colaboradores, 2020; Silva \& Araújo, 2020).

Após contato com humanos, o SARS-CoV-2 mostrou ter um grande potencial de disseminação com o número básico de reprodução (R0) sendo estimado em torno de 2,4 havendo variações a depender da população analisada. Devido à alta infectividade é de grande importância nesse contexto de pandemia que haja uma equipe multidisciplinar que trabalhe de forma colaborativa, uma vez que os serviços de saúde estão sobrecarregados, e o tratamento e manejo clínico de pacientes com COVID19 ainda é cercado de muitas incertezas, tornando-se um desafio em todo o mundo. Dessa forma, o farmacêutico, pode aumentar significativamente o acesso ao cuidado em todos os níveis de atenção em saúde (Bezerra e colaboradores, 2020; Cagnazzo \& Chiari-Andréo, 2020) .

O farmacêutico pode ser encontrado, desde farmácias comunitárias a serviços de saúde mais complexos, e nesse cenário de distanciamento social com acesso restrito a hospitais, este profissional está mais próximo da população. Desse modo as farmácias tornaram-se o primeiro contato da população com o sistema de saúde quer seja em busca de informações ou orientações sobre a doença, quer seja para a aquisição de medicamentos para prevenção ou tratamento no contexto da pandemia (Liu e colaboradores, 2020).

Diante do exposto o objetivo deste estudo foi avaliar a percepção da população sobre o novo coronavírus e sobre a importância do farmacêutico como profissional de saúde atuando na pandemia.

\section{Metodologia}

Trata-se de um estudo descritivo exploratório de abordagem quantitativa, não probabilístico e por conveniência, a coleta de dados foi realizada online via plataforma Google Formulários, no período de outubro a novembro de 2020.

Segundo Marconi e Lakatos (1999) uma pesquisa descritiva trata-se de uma investigação empírica, com o objetivo de conferir hipóteses, delinear um problema ou analisar um fato e pode ser usada para facilitar a elaboração de um questionário ou para servir de base a uma futura pesquisa, ajudando a formular hipóteses. Como método de coleta de dados, utiliza questionários, entrevistas, observação participante (Mattar, 1996).

Foram incluídos no estudo 1.748 pessoas de ambos os sexos, maiores de 18 anos e residentes no Brasil, que aceitaram participar voluntariamente. A amostra foi organizada em três grupos de acordo com a resposta para questão de ${ }^{\circ} 11$ do formulário de pesquisa: “Teve COVID-19?" Sim, representando 11,4\% (200); “não teve”, 59,6\% (1.041), e “não sei”, com 29\% (507). Aqueles que se recusaram a participar, estrangeiros e/ou brasileiros morando no exterior, foram automaticamente excluídos do estudo.

O questionário adotado foi do tipo estruturado não disfarçado, ou seja, o respondente foi informado sobre o objetivo antes do início da pesquisa, através do Termo de Consentimento Livre e Esclarecido e do convite que recebeu para participar. . O questionário tinha 22 questões, fechadas e de múltipla escolha, dividido em duas seções. A primeira com 10 perguntas para a caracterização da amostra (idade, formação, acesso a meios de comunicação, e contato com conteúdos relacionados à COVID19 durante a pandemia), na segunda seção perguntou-se sobre o conhecimento dos participantes sobre a atuação do farmacêutico 
na pandemia, aqui ele foi instigado a responder questões como: "você considera o farmacêutico como profissional da saúde?"; "você sabe como o farmacêutico atuou na pandemia?", dentre outras perguntas, além disso, desses 12 questionamentos feito nessa etapa 4 deles direcionaram o participante a informar sobre como a pandemia afetou seu acesso a medicamentos.

Depois de concluída a introdução do questionário no Google docs, o link do questionário foi enviado via e-mail a pelo menos 100 endereços eletrônicos de pelo menos 100 convidados a participar da pesquisa. As pessoas receberam um e-mail contendo uma pequena explicação sobre a pesquisa, e um link para acesso direto ao questionário. Cada sujeito teve livre escolha para acessar o link e responder ou não ao questionário. Nenhuma das questões foi de caráter obrigatório. Além disso, os usuários do WhatsApp e Facebook receberam um arquivo em PDF e uma imagem em png (usuários do Instagram) contendo uma pequena explicação sobre a pesquisa, e um link para acesso direto ao questionário. Cada sujeito teve a livre escolha para acessar o link e respondê-lo ou não.

Após a finalização da coleta de dados, as respostas válidas foram exportadas para uma planilha do software Microsoft Excel versão 2019 MSO. Para a análise estatística os dados coletados foram registrados numa Planilha do Programa Numbers na versão 6.2.1 (6529) da Apple Inc, posteriormente exportadas para análises no software livre R - versão 3.6.0 GUI 1.70 El Capitan build (7657). Os resultados foram descritos em tabelas de frequência absoluta e percentual, de acordo com os grupos citados anteriormente. As associações entre as variáveis foram obtidas pelo teste Qui-quadrado. O nível de significância estabelecido foi 5\% ( $<0.05$ ). O nível de confiança adotado foi de 95\%. O presente estudo foi submetido ao Comitê de Ética em Pesquisa, CAAE 37991120.3.0000.5214, sendo aprovado sob o parecer nº 4.343.471.

\section{Resultados}

Participaram do estudo 1.748 participantes, sendo 1.128 (64,5\%) mulheres, $612(35 \%)$ homens e 1 (8\%) não se

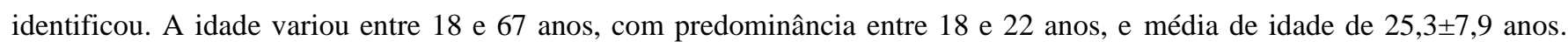
Quanto à cor $993(56,8 \%)$ eram pardos, 435 (24,9) brancos, $272(15,6 \%)$ pretos, 43 (2,5\%) amarelos e $5(0,3 \%)$ indígenas (Tabela 1). 
Research, Society and Development, v. 10, n. 9, e54310918304, 2021

(CC BY 4.0) | ISSN 2525-3409 | DOI: http://dx.doi.org/10.33448/rsd-v10i9.18304

Tabela 1. Perfil sociodemográfico dos entrevistados.

\begin{tabular}{|c|c|c|c|c|c|c|c|c|c|}
\hline & \multicolumn{6}{|c|}{ Tiveram COVID-19 } & \multirow{2}{*}{\multicolumn{2}{|c|}{ Total }} & \multirow{3}{*}{ p-valor } \\
\hline & \multicolumn{2}{|c|}{ Sim } & \multicolumn{2}{|c|}{ Não } & \multicolumn{2}{|c|}{ Não sei } & & & \\
\hline & $\mathbf{N}$ & $\%$ & $\mathbf{N}$ & $\%$ & $\mathbf{N}$ & $\%$ & $\mathbf{N}$ & $\%$ & \\
\hline Sexo & & & & & & & & & 0,325 \\
\hline Feminino & 116 & 58,0 & 684 & 65,7 & 328 & 64,7 & 1128 & 64,5 & \\
\hline Masculino & 83 & 41,5 & 353 & 33,9 & 176 & 34,7 & 612 & 35,0 & \\
\hline Não declarado & 1 & 0,5 & 4 & 0,4 & 3 & 0,6 & 8 & 0,5 & \\
\hline Faixa etária & & & & & & & & & $<0,0001$ \\
\hline $18-22$ & 95 & 47,5 & 518 & 49,8 & 294 & 58,0 & 907 & 51,9 & \\
\hline $23-27$ & 54 & 27,0 & 243 & 23,3 & 125 & 24,7 & 422 & 24,1 & \\
\hline $28-32$ & 19 & 9,5 & 97 & 9,3 & 41 & 8,1 & 157 & 9,0 & \\
\hline $33-37$ & 13 & 6,5 & 66 & 6,3 & 22 & 4,3 & 101 & 5,8 & \\
\hline $38-42$ & 5 & 2,5 & 45 & 4,3 & 12 & 2,4 & 62 & 3,5 & \\
\hline $43-47$ & 4 & 2,0 & 32 & 3,1 & 8 & 1,6 & 44 & 2,5 & \\
\hline $48-52$ & 6 & 3,0 & 25 & 2,4 & 4 & 0,8 & 35 & 2,0 & \\
\hline $53-57$ & 1 & 0,5 & 8 & 0,8 & 1 & 0,2 & 10 & 0,6 & \\
\hline $58-62$ & 3 & 1,5 & 1 & 0,1 & 0 & 0,0 & 4 & 0,2 & \\
\hline 63 ou mais & 0 & 0,0 & 6 & 0,6 & 0 & 0,0 & 6 & 0,3 & \\
\hline Raça & & & & & & & & & 0,390 \\
\hline Pardo & 115 & 57,5 & 588 & 56,5 & 290 & 57,2 & 993 & 56,8 & \\
\hline Branco & 41 & 20,5 & 278 & 26,7 & 116 & 22,9 & 435 & 24,9 & \\
\hline Preta & 36 & 18,0 & 151 & 14,5 & 85 & 16,8 & 272 & 15,6 & \\
\hline Amarelo & 7 & 3,5 & 21 & 2,0 & 15 & 3,0 & 43 & 2,5 & \\
\hline Indigena & 1 & 0,5 & 3 & 0,3 & 1 & 0,2 & 5 & 0,3 & \\
\hline
\end{tabular}

*Qui-quadrado. Fonte: Autores.

Com relação à opinião dos respondentes sobre grupos de risco, $92 \%$ dos participantes concordaram totalmente que idosos, hipertensos e diabéticos faziam parte do grupo de risco para a COVID-19. Quanto a ser grupo com maior número de óbitos $51,2 \%$ dos participantes do estudo concordaram totalmente que idosos, diabéticos e hipertensos foram os que mais morreram em decorrência do coronavírus, enquanto 1,4\% discordaram totalmente. Questionados sobre qual o sexo foi o mais contaminado pelo coronavírus, $74,7 \%$ citaram o masculino e $25,3 \%$, o feminino (Tabela 2). 
Research, Society and Development, v. 10, n. 9, e54310918304, 2021

(CC BY 4.0) | ISSN 2525-3409 | DOI: http://dx.doi.org/10.33448/rsd-v10i9.18304

Tabela 2. Percepção da população acerca da epidemiologia da COVID-19.

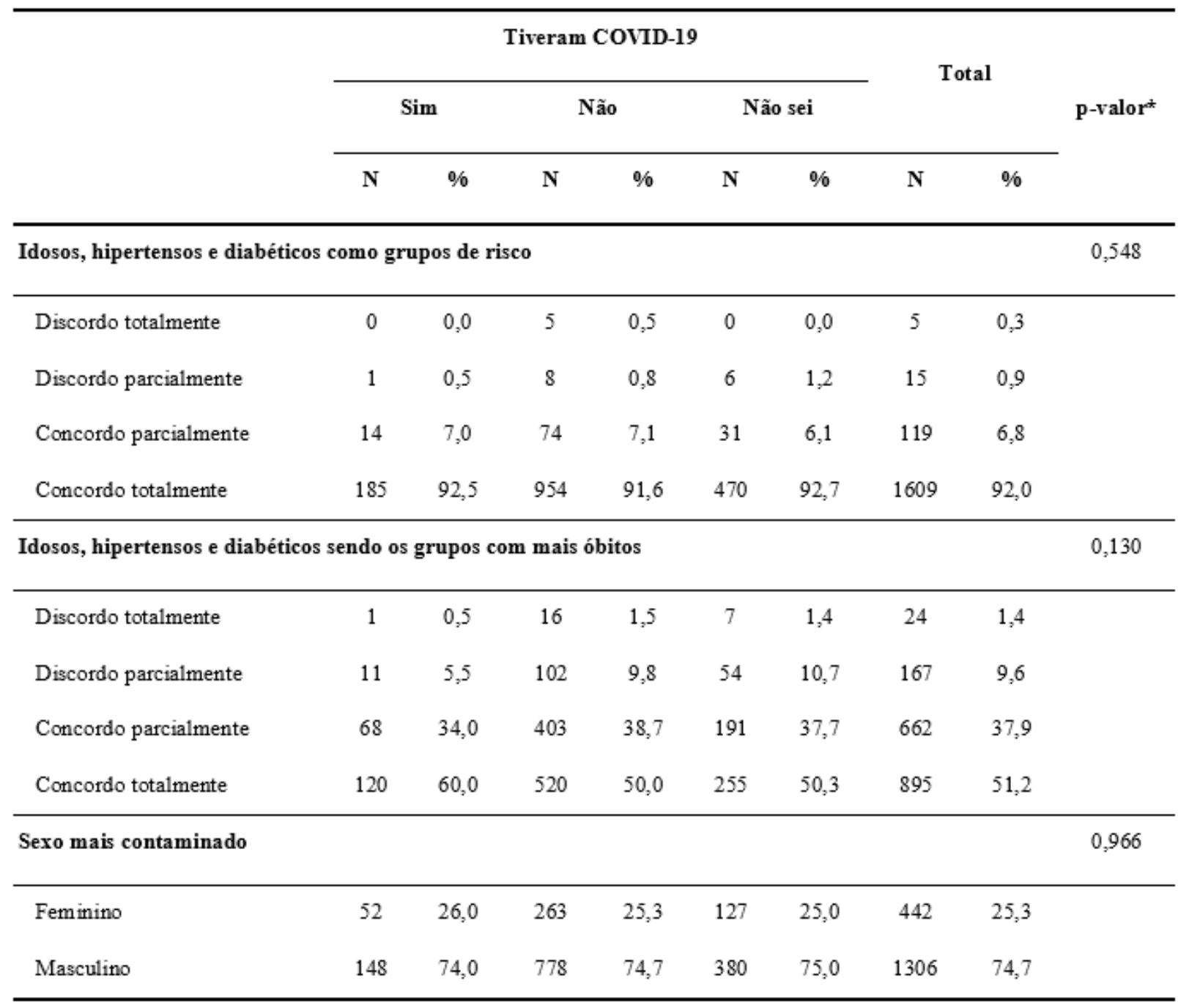

*Qui-quadrado. Fonte: Autores.

Os participantes responderam se acreditavam nos estudos científicos divulgados sobre os tratamentos (pergunta de $\mathrm{n}^{\circ}$ 21) para o SARS-CoV-2 e se fariam uso ou mesmo aconselhariam familiares e/ou amigos a utilizarem tratamentos que não são aconselhados pelas autoridades de saúde (pergunta de $n^{\circ} 22$ ) (Tabela 3). 
Research, Society and Development, v. 10, n. 9, e54310918304, 2021

(CC BY 4.0) | ISSN 2525-3409 | DOI: http://dx.doi.org/10.33448/rsd-v10i9.18304

Tabela 3. Informações sobre tratamentos e uso de medicamentos na pandemia.

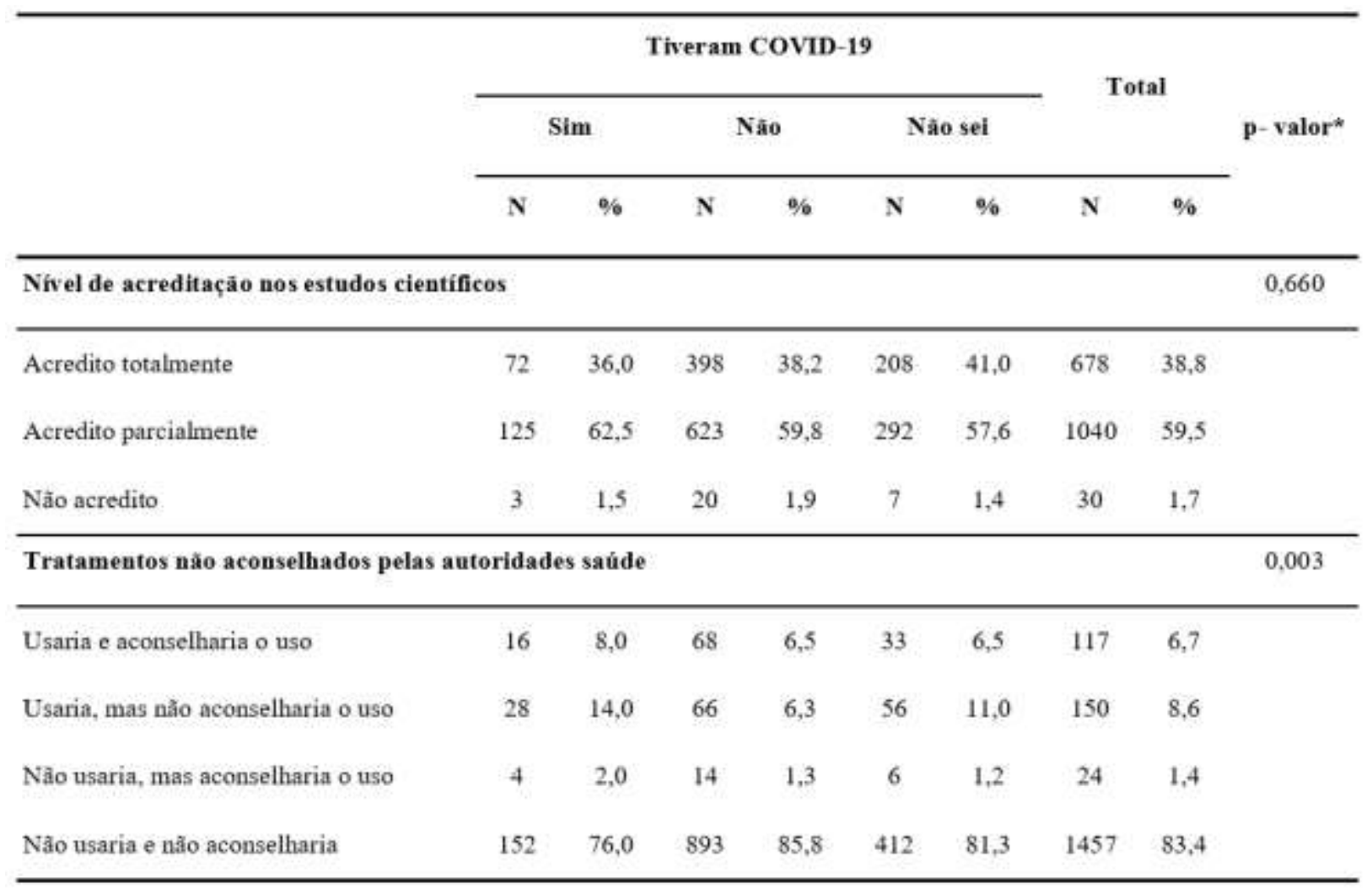

*Qui-quadrado. Fonte: Autores.

Cerca de $60 \%$ dos respondentes disseram acreditar apenas parcialmente nos estudos científicos divulgados, enquanto 38,8\% responderam acreditar totalmente. Não sendo observado resultado estatisticamente significativo $(\mathrm{p}=0,660)$ entre os grupos. Ao passo que $83,4 \%$ não usaria e não aconselharia o uso de tratamentos que não são recomendados pelas autoridades de saúde (p=0,003). Entre aqueles que tiveram COVID-19, a maioria (76\%) afirmou que não faria uso e não aconselharia tratamentos sem respaldo das autoridades de saúde. Todavia, comparando-se os grupos, $14 \%$ das pessoas do grupo "sim" fariam uso de algum medicamento não aconselhado, observando-se uma diminuição nessa porcentagem nos outros grupos (Tabela 3).

Os resultados apresentados na Tabela 4 evidenciam o impacto da pandemia sobre o acesso a medicamentos em farmácias e drogarias comunitárias, e em farmácias do Sistema Único de Saúde - SUS. 
Tabela 4. Acesso a medicamentos na pandemia do novo coronavírus.

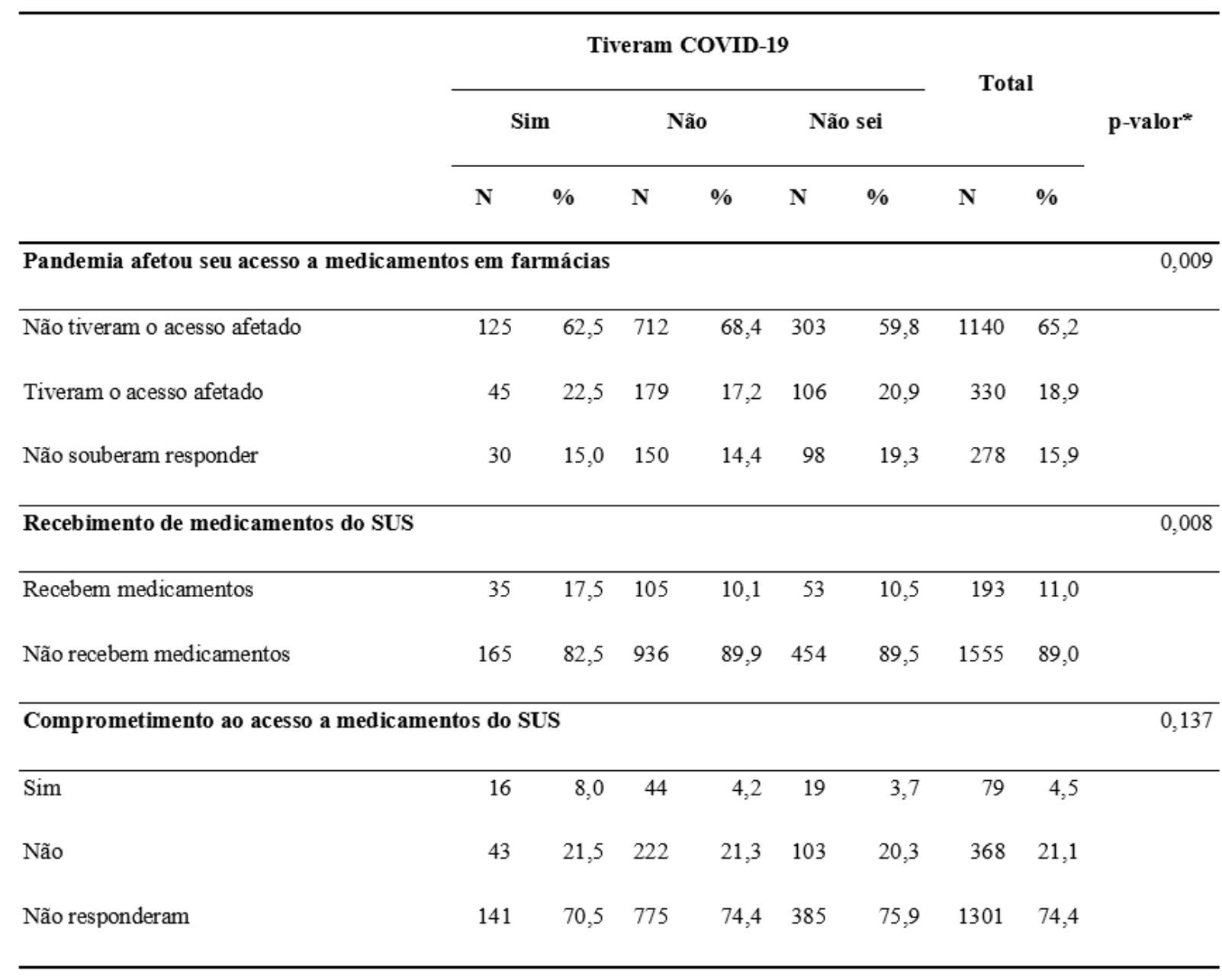

*Qui-quadrado. Fonte: Autores.

Dos 1.748 participantes, 65,2\% não tiveram o acesso a medicamentos afetado e 18,9\% responderam que sim (Tabela 4). A amostra estudada quando infectada pelo SARS-Cov-2 durante a pandemia não teve dificuldade no acesso a medicamentos $(\mathrm{p}=0,009)$. Questionados sobre o recebimento de medicamentos pelo SUS 89\% não recebiam medicamentos pelo SUS ( $\mathrm{p}=$ 0,008), demonstrando que a maioria dos participantes do estudo que "não tiveram COVID-19" não recebiam nenhum tipo de medicamento pelo SUS, assim como também os que "tiveram COVID-19".

Quanto ao reconhecimento do farmacêutico, 99\% dos entrevistados no grupo que teve COVID-19 o reconheceram ( $\mathrm{p}=$ 0,018) como profissional da saúde. Com relação a ser considerado profissional da linha de frente no combate ao coronavírus, $53,7 \%$ dos entrevistados concordaram totalmente que o farmacêutico faz parte da linha de frente. Referente a atuação desse profissional na pandemia, 43,1\% dos participantes disseram saber como o farmacêutico atuou na pandemia (Tabela 5). 
Tabela 5. Perceção da população sobre o farmacêutico na pandemia.

\begin{tabular}{|c|c|c|c|c|c|c|c|c|c|}
\hline & \multicolumn{6}{|c|}{ Tiveram COVID-19 } & \multirow{2}{*}{\multicolumn{2}{|c|}{ Total }} & \multirow{3}{*}{ p-valor* } \\
\hline & \multicolumn{2}{|c|}{ Sim } & \multicolumn{2}{|c|}{ Não } & \multicolumn{2}{|c|}{ Não sei } & & & \\
\hline & $\mathbf{N}$ & $\%$ & $\mathbf{N}$ & $\%$ & $\mathbf{N}$ & $\%$ & $\mathbf{N}$ & $\%$ & \\
\hline \multicolumn{7}{|c|}{ Reconhece o farmacêutico como profissional da saúde } & & & 0,018 \\
\hline Sim & 198 & 99,0 & 994 & 95,5 & 494 & 97,4 & 1686 & 96,5 & \\
\hline Não & 2 & 1,0 & 47 & 4,5 & 13 & 2,6 & 62 & 3,5 & \\
\hline \multicolumn{9}{|c|}{ Farmacêutico na linha de frente da COVID-19 } & 0,441 \\
\hline Discordo totalmente & 3 & 1,5 & 17 & 1,6 & 7 & 1,4 & 27 & 1,5 & \\
\hline $\begin{array}{l}\text { Discordo } \\
\text { parcialmente }\end{array}$ & 22 & 11,0 & 114 & 11,0 & 60 & 11,8 & 196 & 11,2 & \\
\hline $\begin{array}{l}\text { Concordo } \\
\text { parcialmente }\end{array}$ & 55 & 27,5 & 368 & 35,4 & 164 & 32,3 & 587 & 33,6 & \\
\hline Concordo totalmente & 120 & 60,0 & 542 & 52,1 & 276 & 54,4 & 938 & 53,7 & \\
\hline \multicolumn{9}{|c|}{ Atuação do farmacêutico na pandemia } & 0,364 \\
\hline Sim & 97 & 48.5 & 448 & 43,0 & 208 & 41,0 & 753 & 43,1 & \\
\hline Não & 51 & 25,5 & 310 & 29,8 & 146 & 28,8 & 507 & 29,0 & \\
\hline Talvez & 52 & 26,0 & 283 & 27,2 & 153 & 30,2 & 488 & 27,9 & \\
\hline
\end{tabular}

*Qui-quadrado. Fonte: Autores.

De acordo com resultados os infectados pelo coronavírus foram os que mais relataram serem atendidos por farmacêuticos durante a pandemia, demonstrando que houve uma correlação estatisticamente significativa ( $\mathrm{p}<0,0001)$ entre “ter COVID-19” e ter sido atendido por farmacêutico, assim como também houve correlação significativa para a variável "busca por orientação farmacêutica", uma vez que o grupo que teve COVID-19 foi o que mais buscou por orientação desse profissional durante a pandemia $(\mathrm{p}<0,0001)$. 
Tabela 6. Atendimento por farmacêutico na pandemia.

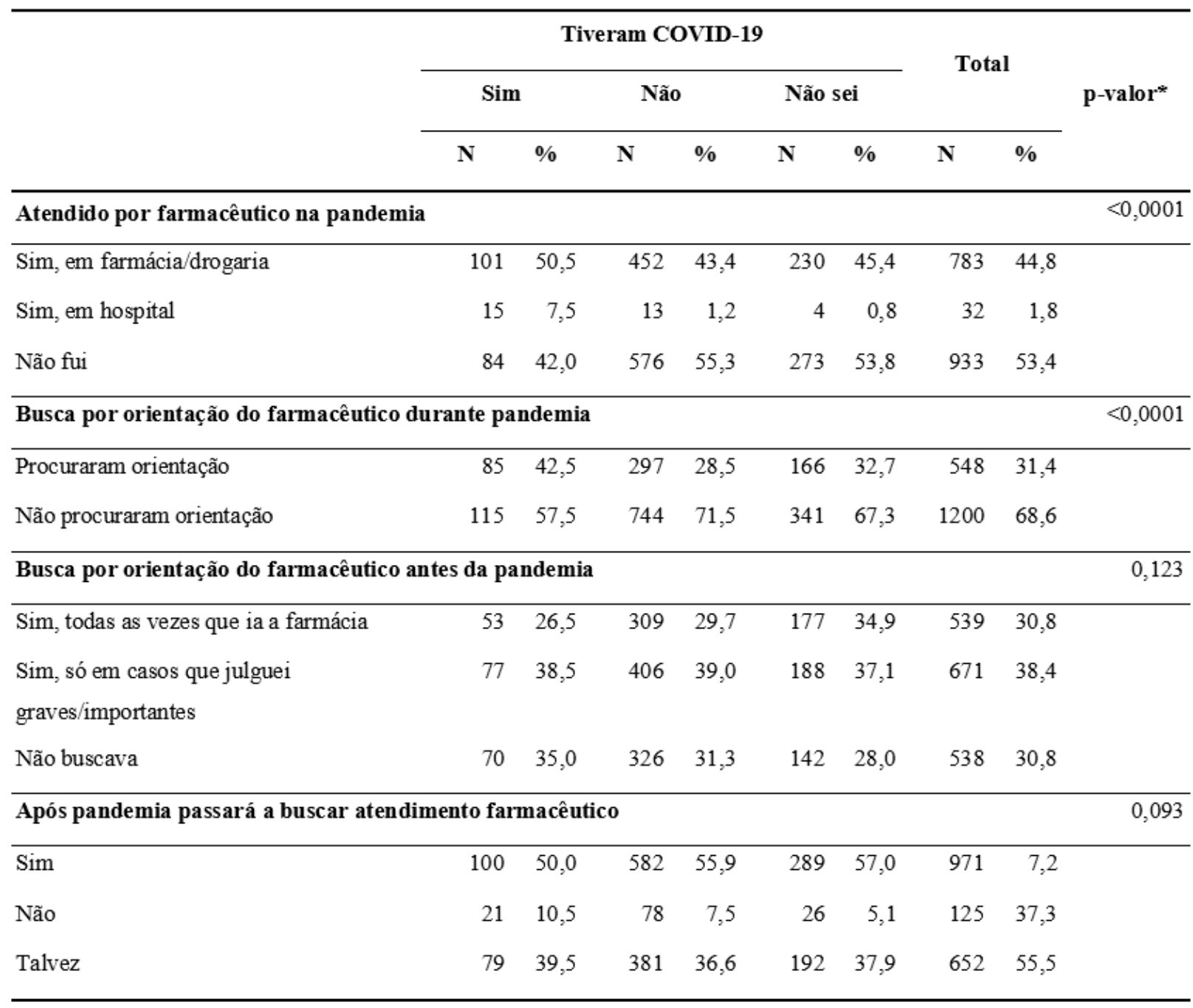

*Qui-quadrado. Fonte: Autores.

Quando perguntados se buscarão orientação do farmacêutico na pós-pandemia 55,5\% afirmou que talvez busque, enquanto apenas 7,2\% afirmou que passará a buscar atendimento com esse profissional (Tabela 6).

\section{Discussão}

Dados divulgados pelo Boletim Epidemiológico do Ministério da Saúde (semana epidemiológica 51 de 13/12 a 19/12) apontaram que a faixa etária mais acometida pela doença foi a de 60 a 69 anos (Brasil, 2015), entretanto em nossos achados a média de idade de 25,3ะ7,9 anos. Segundo dados divulgados pela Pesquisa Nacional por Amostra de Domicílios Contínua PNAD Contínua (2018) a maioria dos usuários das redes sociais e da internet são jovens de 20-24 anos (91\%), isso pode ser percebido pela diferença entre a nossa média de idade e os divulgados pelo Ministério da Saúde (IBGE, 2019).

Estudos transversais com aplicação de questionários on line avaliando os impactos da COVID-19 observaram uma maior participação de mulheres entre os respondentes com prevalência de cerca de 70\% e 83\% (Bezerra e colaboradores, 2020; Duarte e colaboradores, 2020). Esse maior predomínio pode estar relacionado ao fato da população brasileira ter maior prevalência do gênero feminino em relação ao masculino (IBGE, 2020). Outro fator seria o maior uso da internet pelas mulheres $(75,7 \%)$ em contraponto aos homens $(73,6 \%)$ (IBGE, 2019). 
Com relação à opinião dos respondentes sobre grupos de risco, 92\% dos participantes concordaram totalmente que idosos, hipertensos e diabéticos faziam parte do grupo de risco para a COVID-19. Quanto a ser grupo com maior número de óbitos $51,2 \%$ dos participantes do estudo concordaram totalmente que idosos, diabéticos e hipertensos foram os que mais morreram por conta do coronavírus. Os resultados obtidos corroboram com estudos que observaram que a proporção de pacientes com graus de maior gravidade para pneumonia por COVID-19 foi significativamente maior no grupo de idosos ( $\mathrm{p}<0,05$ ), com a população idosa representando $85 \%$ dos casos de óbitos registrados até o dia 3 de abril de 2020 no Brasil (Liu e colaboradores, 2020; Silva \& Oliveira, 2020).

Estudo realizado por Niquini e colaboradores (2020) revelou que 33,6\% dos adultos com mais de 60 anos com diagnóstico de diabetes mellitus foram hospitalizados em decorrência da COVID-19, a mesma tendência foi observada para idosos com cardiopatias, no qual 57,4\% foram hospitalizados. Dados do Boletim Epidemiológico da Secretária de Vigilância em Saúde apresentaram o mesmo cenário, indivíduos que evoluíram para óbito tinham alguma comorbidade e apresentavam 60 anos ou mais. Além disso, 65,5\% dos óbitos apresentavam pelo menos uma comorbidade ou fator de risco para a doença, sendo cardiopatia e diabetes mellitus (DM) a condição mais frequente (Brasil, 2015).

O SARS-CoV-2 afeta a parte endócrina do pâncreas com consequente hiperglicemia, possivelmente pela superexpressão de ACE-2 pelas células das ilhotas pancreáticas, estas responsáveis pela síntese de insulina, que controla a glicemia (Yang e colaboradores, 2010). Dessa forma, pessoas com DM têm aumento na expressão de ACE-2, o que pode ser um fator predisponente à infecção pelo vírus (Singh e colaboradores, 2020).

Uma metanálise envolvendo 33 estudos e 16.003 participantes mostrou que pessoas com DM e COVID-19 têm maior risco de severidade, com razão de chance de 2,75 (IC 95\%:2,09 e 3,62; p <0,01) quando comparados àqueles com COVID-19 e sem DM; e têm maior risco de mortalidade, com uma razão de chance de 1,90 (IC 95\%:1,37 e 2,64; p <0,01). A prevalência de DM em indivíduos com COVID-19 foi de 9,8\% (IC 95\%:8,7\% e 10,9\%), após ajuste de heterogeneidade (Kumar e colaboradores, 2020). Esta associação, entretanto, ainda é tema de debate em busca por novas evidências científicas.

Dentre as comorbidades associadas à COVID-19 as cardiopatias ocupam a posição de maior destaque. Estudos apontam que cardiopatas, assim como aqueles acometidos por outras doenças crônicas, possuem chance aumentada de apresentar um pior prognóstico quando infectados pelo novo coronavírus (Costa e colaboradores, 2020; Rente, Uezato Jr \& Uezato, 2020). Dados da American College of Cardiology em 2020, corroboram com esses achados quando afirmam que os níveis de hospitalização em pessoas com cardiopatias e COVID-19 chegam a ser 50\% maiores que com aqueles sem cardiopatias (American College of Cardiology, 2020).

Analisando-se as respostas dos entrevistados nesse estudo sobre o sexo mais acometido pelo novo coronavírus com autores que pesquisaram a epidemiologia da COVID-19 no país, observou-se que o masculino foi o mais contaminado, representando 60\% das hospitalizações por SRAG por COVID-19 (Niquini e colaboradores, 2020). Dados do Ministério da Saúde evidenciaram o mesmo panorama, com 56,1\% dos casos de SRAG por COVID-19 notificados em homens, de modo que eles também foram responsáveis pela maioria dos óbitos (57,5\%) (Brasil, 2015).

No presente estudo o gênero feminino foi o menos infectado pelo novo coronavírus, entretanto Chen e colaboradores (2020) relataram que a contaminação por COVID-19 parece ter uma predileção pelo sexo. Historicamente, em epidemias anteriores de SARS e MERS, os homens também foram mais propensos a serem contaminados que as mulheres, isso pode ter associação com o importante papel que os cromossomos $\mathrm{X}$ da mulher e os hormônios sexuais desempenham no sistema imunológico do corpo, além também do senso de autocuidado ser superior em relação aos homens (Jaillon, Berthenet \& Garlanda, 2019). Ademais, culturalmente o homem se vê como um ser invulnerável, fato que contribui para que ele negligencie seu autocuidado e se exponha mais a situações de risco (Gomes, Nascimento \& Araújo, 2007). 
Além da epidemiologia, o estudo também quis saber sobre os aspectos relacionados ao uso de medicamentos pela população, bem como, avaliar a opinião desses sobre as evidências científicas em torno desses produtos utilizados para o tratamento da COVID-19, visto que o uso de algumas drogas como, Hidroxicloroquina (HCQ), Cloroquina, Ivermectina, Azitromicina, Prednisona, dentre outras, tornaram-se durante a pandemia assunto recorrente tanto no meio científico, na TV aberta e nas redes sociais.

Quando perguntados se acreditavam em estudos científicos divulgados sobre os tratamentos para o SARS-CoV-2 e se fariam uso ou mesmo aconselhariam familiares e/ou amigos a utilizarem tratamentos não são aconselhados por autoridades de saúde, apenas 38,8\% dos participantes respondeu acreditar na ciência e 83,4\% declarou que não aconselharia ou mesmo recomendaria uso de medicamento sem o aval de especialistas. A pandemia impôs grandes desafios a todos os países, vários setores da sociedade civil, e em especial à comunidade científica, que respondeu de forma rápida e eficiente, com o desenvolvimento de novas vacinas, métodos diagnósticos, e desenvolvimento de estudos na busca por novos tratamentos que fossem seguros e ativos contra o vírus (Ferreira \& Andricopulo, 2020). Todavia, até o presente momento ainda não há um medicamento, fármaco, princípio ativo ou fórmula farmacêutica cientificamente eficaz contra o SARS-Cov-2.

No entanto, apesar de resultados favoráveis para o nível de acreditação e não uso de medicamentos não recomendados, tem-se observado uma tendência de negacionismo nas evidências científicas, que se agravou nessa pandemia, levando a aceitação de intervenções sem validações científicas como a divulgação e exaltação de terapêuticas de eficácia não comprovada (Caponi, 2020; Guimarães \& Carvalho, 2020). Observa-se essa tendência no aumento nas pesquisas via internet pelos medicamentos relatados bem como a escassez desses fármacos, mortes e intoxicações (Luccheta \& Mastroianni, 2019). Nesse cenário o farmacêutico como profissional mais próximo da população em farmácias comunitárias tem papel fundamental na educação em saúde direcionando-a para medidas de prevenção e controle da infecção pelo novo coronavírus, orientando a população sobre os possíveis tratamentos e contribuindo para a diminuição do uso indiscriminado de medicamentos (Pharmaceutical Society of Australia, 2021).

No Brasil, a escassez de fármacos veio não somente com o incentivo ao uso de medicamentos sem comprovação terapêutica, mas também com o aumento mundial no consumo de produtos para a saúde,dificuldades de importação, custos exorbitantes da mataéria-prima, pagamentos antecipados obrigatórios, descumprimento de quantidades acordadas e prazos de entrega; afetando, portanto, o fornecimento de fármacos essenciais no tratamento da COVID-19 como os sedativos (fentanil e midazolam) e os agentes neuromusculares (suxamethônio, atracúrio) (Badreldin \& Atallah, 2020; Martins e colaboradores, 2020).

Já quanto ao acesso a medicamentos em farmácias e/ou drogarias para o tratamento da COVID-19 os participantes do estudo declararam pouca dificuldade na aquisição de medicamentos durante a pandemia. Nesse cenário, vale destacar que durante a pandemia aumentou o padrão de consumo de medicamentos no Brasil em farmácias e drogarias, estimulado principalmente pela alardeamento do "tratamento precoce" e do "Kit COVID". Segundo levantamento do Sindicato da Indústria de Produtos Farmacêuticos (Sindusfarma), esses medicamentos representaram uma movimentação nos caixas das empresas farmacêuticas nacionais cifras próximas a R \$ 500 milhões somente no ano de 2020. Isso permite levantar a hipótese de aumento no risco relativo do aparecimento de Reações Adversas a Medicamentos (RAM) associadas principalmente a hidroxicloroquina e cloroquina (Scaramuzzo, 2020; Melo e colaboradores, 2021).

Em relação aos infectados pelo novo coronavírus, o não comprometimento no acesso aos medicamentos para tratar a doença foi graças às medidas adotadas pelo SUS, através da Agência Nacional de Vigilância Sanitária (ANVISA), que enquadrou medicamentos, a exemplo da Cloroquina e HCQ, no Anexo I da Portaria 344/1998, passando esses a serem considerados de controle especial, além disso, medicamentos como a Ivermectina passaram a ser vendidos com retenção de receita (Anvisa, 2020). Outra importante medida tomada no âmbito do SUS para evitar o desabastecimento foi a publicação da 
Resolução da Diretoria Colegiada (RDC) 389/2020, que dispõe sobre os requisitos temporários para caracterização e verificação do risco de redução da oferta de medicamentos durante a pandemia do novo coronavírus (Pfarma, 2020). Assim, apesar da dificuldade na aquisição de IFA, a população não ficou desassistida e por isso não foi verificada uma grande porcentagem de pessoas com acesso afetado a medicamentos nesse estudo, principalmente daqueles testados para o SARS-Cov-2.

Os farmacêuticos, detentores de conhecimentos relacionados a serviços gerenciais, é também importante no gerenciamento da escassez de fármacos, pois através do ciclo da Assistência Farmacêutica (seleção, programação, aquisição, distribuição e armazenamento) são capazes de gerenciar crises no abastecimento e propor soluções de forma integrada com os setores dentro da Rede de Atenção à Saúde (Brasil, 2020). Mesmo com as grandes contribuições que o farmacêutico pode agregar na equipe multidisciplinar e oferecendo assistência à saúde são raramente mencionados por organizações mundiais, agências de saúde, mídia e outrem que reconhecem apenas médicos e enfermeiros por seus esforços na linha de frente da pandemia (Goff e colaboradores, 2020).

O farmacêutico encontra-se mais próximo da população na farmácia comunitária, que no atual contexto é o local onde ele realiza o gerenciamento de problemas de saúde autolimitados dos sintomas leves causados pelo novo coronavírus (Dzingirai e colaboradores, 2020). Com relação a ser considerado profissional da linha de frente no combate à COVID-19 53,7\% dos entrevistados concordaram totalmente que o farmacêutico faz parte da linha de frente, e quanto à sua atuação $43,1 \%$ dos participantes disseram saber como ele atuou na pandemia.

O reconhecimento de grande parte dos entrevistados sobre a atuação do farmacêutico na linha de frente da pandemia revela que atualmente o farmacêutico não é mais considerado apenas um especialista em medicamentos, mas um profissional relevante na equipe de saúde e na divulgação de informação de alta qualidade educando, aconselhando e capacitando os cidadãos para terem autonomia no cuidado de sua saúde (Urick \& Meggs, 2019). Entretanto, outra parcela dos respondentes desconhecia total ou parcialmente a forma como o farmacêutico atuou na pandemia, mesmo considerando-o um profissional na linha de frente, portanto nota-se que há ainda a necessidade da classe em empreender maiores esforços para que sua atuação seja, sobretudo entendida pela população, mídia e governantes, para elevar o nível das expectativas do público sobre como os farmacêuticos contribuem para o uso da qualidade dos medicamentos não só durante a pandemia e situações crônicas (Cheong e colaboradores, 2020; Zheng e colaboradores, 2021; Bragazzi e colaboradores, 2020).

De acordo com nossos achados os infectados pelo coronavírus foram os que mais relataram serem atendidos por farmacêuticos durante a pandemia. Já com relação a procura pelo atendimento do farmacêutico antes da pandemia, a maioria só buscou por atendimento nos casos em que julgou grave e quando perguntados se buscarão a orientação farmacêutica na póspandemia a maioria afirmou que talvez busque (55,5\%), enquanto apenas 7,2\% afirmou que passará a buscar atendimento com esse profissional, reforçando o entendimento de que a classe farmacêutica precisa ser mais reconhecida pelos serviços de grande relevância prestados à sociedade, não só em tempos de calamidade na saúde, mas ao longo dos anos de atuação profissional promovendo o uso seguro de medicamentos, autonomia no cuidado, melhorando a segurança do paciente e a saúde pública (Agomo, 2012; Marwitz, 2020).

Vale ressaltar ainda que é ele também a última barreira entre o indivíduo e a farmacoterapia, evitando que ocorram erros de dispensação, interação medicamentosa e acima de tudo uso irracional de medicamentos. 


\section{Conclusão}

Constatou-se que o farmacêutico foi reconhecido como profissional de saúde e atuante na linha de frente contra o novo coronavírus. Todavia, vale destacar que o serviço de orientação farmacêutica quando procurado foi somente para os casos considerados graves ou importantes.

É imprescindível que as organizações farmacêuticas, além de defender legislações e políticas que apoiem e incentive a prática da farmácia em equipes multidisciplinares e farmácias comunitárias, definam e divulguem claramente as atividades clínicas dos farmacêuticos, fazendo com que a população procure por esse profissional e tenha confiança no seu trabalho e nas informações por ele prestadas.

\section{Referências}

Ashour, H. M., Elkhatib, W. F., Rahman, M., \& Elshabrawy, H. A. (2020). Insights into the Recent 2019 Novel Coronavirus (SARS-CoV-2) in Light of Past Human Coronavirus Outbreaks. Pathogens. 9 (3), 186.

Dzingirai, B., Matyanga, C. M. J., Mudzviti, T., Siyawamwaya, M., \& Tagwireyi, D. (2020). Riscos para os farmacêuticos comunitários e o pessoal da farmácia durante a pandemia COVID-19: perspectivas de um país de baixa renda. J of Pharm Policy and Pract. 13(42).

Silva, L. M. C., \& Araújo, J. L. (2020). Clinical and community pharmacist's role in the COVID-19 pandemic. Research, Society and Development. 9 (7):1-14. Bezerra, A. C. V., Silva, C. E. M., Soares, F. R. G., \& Silva, J. A. M. (2020). Fatores associados ao comportamento da população durante o isolamento social na pandemia de COVID-19. Ciência \& Saúde Coletiva. 25(1): 2411-2421.

Cagnazzo, D. I. T. O., \& Chiari-Andréo, B. G. (2020). COVID - 19: cuidados farmacêuticos durante a pandemia. Rev. Bras. Multi. 23(1).

Liu, K., Chen, Y., Lin, R., \& Han, K. (2020). Clinical features of COVID-19 in elderly patients: A comparison with young and middle-aged patients. J Infect. 80 (6): $14-18$

Brasil. Presidência da República, Secretaria de Comunicação Social. Pesquisa brasileira de mídia 2015: hábitos de consumo de mídia pela população brasileira. Brasília (DF), Secretaria de Comunicação Social. (2015). https://www.researchgate.net/publication.

IBGE - Instituto Brasileiro de Geografia e Estatística. Pesquisa Nacional por Amostra de Domicílios Contínua. Rio de Janeiro (RJ). (2019).

Duarte, M. Q., Santo, M. A. S., Lima, C. P., Giordani, J. P., \& Trentini, C. M. (2020). COVID-19 e os impactos na saúde mental: uma amostra do Rio Grande do Sul, Brasil. Ciência \& Saúde Coletiva. 25(9): 3401-3411.

IBGE - Instituto Brasileiro de Geografia e Estatística. Pesquisa Nacional por Amostra de Domicílios Contínua. Rio de Janeiro (RJ). PNAD COVID19: Resultado mensal novembro/2020.

Silva, D. F., \& Oliveira, M. L. C. (2020). Epidemiologia da COVID-19: comparação entre boletins epidemiológicos. Com. Ciências Saúde. 31(1): 61-74.

Niquini, R. P., Lana, R. M., Pacheco, A. G., Cruz, O. G., Coelho, F. C., Carvalho, L. M., Villela, D. A. M., Gomes, M. F. C., \& Bastos, L. S. (2020). SRAG por COVID-19 no Brasil: descrição e comparação de características demográficas e comorbidades com SRAG por influenza e com a população geral. Cad. Saúde Pública. 36(7): e00149420.

Yang, J. K., Lin, S. S., Ji, X. J., \& Guo, L. M. (2010). Binding of SARS coronavirus to its receptor damages islets and causes acute diabetes. Acta Diabetol. 47 (3): 193-9.

Singh, A. K., Gupta, R., Ghosh, A., \& Misra, A. (2020). Diabetes in COVID-19: Prevalence, pathophysiology, prognosis and practical considerations. Diabetes Metab Syndr. 14(4): 303-310.

Kumar, A., Arora, A., Sharma, P., Anikhindi, S. A., Bansal, N., Singla, V., Khare, S., Srivastava, A. (2020). Is diabetes mellitus associated with mortality and severity of COVID-19? A meta-analysis. Diabetes Metab Syndr. (4):535-545.

Costa, J., Silveira, J., Santos, J., Nogueira, S., Pereira, P. (2020). Implicações Cardiovasculares em Pacientes Infectados com Covid-19 e a Importância do Isolamento Social para Reduzir a Disseminação da Doença. Arquivos Brasileiros de Cardiologia. 114 (5): $834-838$.

Rente, A., Uezato-Junior, D., Uezato, K. (2020). Coronavírus e o Coração - Um Relato de Caso sobre a Evolução da COVID-19 Associado à Evolução Cardiológica. Arq. Bras. Cardiol. 114(5): 839-842.

American College of C. (2020). COVID-19 Clinical Guidance For the Cardiovascular Care Team. https://www.acc.org.

Chen, N., Zhou, M., Dong, X., Qu, J., Gong, F., Han, Y., Qiu, Y., Wang, J., Liu, Y., Wei, Y., Xia, J., Yu, T., Zhang, X., \& Zhang. L. (2020). Epidemiological and clinical characteristics of 99 cases of 2019 novel coronavirus pneumonia in Wuhan, China: a descriptive study. Lancet. 395(10223):507-513.

Jaillon, S., Berthenet, K., \& Garlanda, C. (2020). Sexual dimorphism in innate immunity. Clin Rev Allergy Immunol. 56(3): 308-321.

Gomes, R., Nascimento, E. F., \& Araújo, F. C. (2007). Por que os homens buscam menos os serviços de saúde do que as mulheres? As explicações de homens com baixa escolaridade e homens com ensino superior. Cad Saúde Pública. 23 (3): 565-574. 
Ferreira, L. G., Andricopulo, A. D. (2020). Covid-19: Small-molecule clinical trials landscape. Curr. Top. Med. Chem. 20 (18):1577-80.

Caponi, S. (2020). COVID-19 no Brasil: entre o negacionismo e a razão neoliberal. Estud. av. 34(99): 209-224.

Guimarães, A. S., \& Carvalho, W. R. G. (2020). Desinformação, negacionismo e automedicação: a relação da população com as drogas “milagrosas” em meio à pandemia da COVID-19. Inter Am J Med Health. 3: e. 202003053.

Luccheta, C. R., \& Mastroianni, P. C. (2019). Rational use of chloroquine and hydroxychloroquine in times of COVID-19. Revista de Ciências Farmacêuticas Básica e Aplicada. 40: e643. Pharmaceutical Society of Australia (PSA). Coronavirus disease (COVID-19) information for pharmacists.

Badreldin, H. A., \& Atallah, B. (2020). Global drug shortages due to COVID-19: Impact on patient care and mitigation strategies. Res. Social Adm. Pharm. 17: 1946-1949.

Martins, M. A. P., Medeiros, A. F., Almeida, C. D. C., \& Reis., A. M. M. (2020). Preparedness of pharmacists to respond to the emergency of the COVID-19 pandemic in Brazil: a comprehensive overview. Drogas Ther Perspect. 1-8.

Scaramuzzo, M. (2020). Venda de remédios do 'kit covid' movimenta R $\$ 500 \mathrm{mi}$ em 2020. https://valor.globo.com/empresas/noticia/venda-de-remedios-do-kitcovid-movimenta-r-500-mi-em-2020.ghtml

Marconi, M. A., Lakatos, E. (1999) Técnicas de Pesquisa. São Paulo: Atlas. 4 ed

Marwitz, K. K. (2020). The pharmacist's active role in combating COVID-19 medication misinformation. J Am Pharm Assoc. S1544-3191(20)30548-3.

Mattar, F. N. (1996). Pesquisa de marketing: edição compacta. São Paulo: Atlas. Ed compacta.

Melo, J. R. R., Duarte, E. C., Moraes, M. V., Fleck, K., Arrais, S. D. (2021). Automedicação e uso indiscriminado de medicamentos durante a pandemia da COVID-19. Cad. Saúde Pública. 37 (4).

Anvisa - Agência Nacional de Vigilância Sanitária. (2020). Hidroxicloroquina e cloroquina viram produtos controlados. Brasil. [citado 2021 Jan 06]. Pfarma. Anvisa publica resolução sobre desabastecimento de medicamentos durante a pandemia por coronavírus. https://pfarma.com.br/noticia-setorfarmaceutico/legislacao-farmaceutica.

Brasil. Ministério da Saúde, Secretaria de Vigilância em Saúde. Boletim Epidemiológico Especial: Doença pelo Coronavírus COVID-19. Brasília (DF), Secretaria de Vigilância em Saúde. (2020). https://www.gov.br/saude/pt-br/media.

Goff, D. A., Ashiru-Oredope, D., Cairns, K. A., Eljaaly, K., Gauthier, T. P., Langford, B. J., Mahmoud, S.F., Messina, A. P., Michael, U. C., Saad, T., Schellack, N. (2020). Global contributions of pharmacists during the COVID-19 pandemic. J Am Coll Clin Pharm. 1-13.

Urick, B. Y., \& Meggs, E. V. (2019). Towards a Greater Professional Standing: Evolution of Pharmacy Practice and Education, 1920-2020. Pharmacy (Basel). 7(3): 98 .

Cheong, M. W., Btock, T., Karwa, R., \& Pastakia, S. (2020). COVID-19 e farmácia clínica em todo o mundo - a acordar e um chamado à ação. J Am Coll Clin Pharm. 3: 860-863.

Zheng, S. G., Yang, L., Zhou, P. X., Li, H. B., Liu, F., \& Zhao, R. S. (2021). Recommendations and guidance for providing pharmaceutical care services during COVID-19 pandemic: A China perspective. Research in Social and Administrative Pharmacy. 17: 1819-1824.

Bragazzi, N. L., Mansour, M., Bonsignore, A., \& Ciliberti, R. (2020). The Role of Hospital and Community Pharmacists in the Management of COVID-19: Towards an Expanded Definition of the Roles, Responsibilities, and Duties of the Pharmacist. Pharmacy. 8 (140): 1-15.

Agomo, C. O. (2012). The role of community pharmacists in public health: A scoping review of the literature. Journal of Pharmaceutical Health Services Research. 3 (1): 25-33. 